\title{
Factores genéticos que inciden en la resistencia a enfermedades infecciosas en salmónidos y su aplicación en programas de mejoramiento
}

\author{
Genetic factors involved in resistance to infectious diseases in salmonids \\ and their application in breeding programmes
}

\author{
JM Yáñez, V Martínez* \\ Unidad de Genómica y Mejoramiento Genético Animal, Laboratorio de Investigaciones en Biotecnología y Genómica Animal \\ (FAVET-INBIOGEN), Facultad de Ciencias Veterinarias y Pecuarias, Universidad de Chile, Santiago, Chile
}

\begin{abstract}
SUMMARY
The control of infectious diseases is essential in the success of salmon aquaculture. Genetic improvement of disease resistance may provide a feasible and sustainable option for the management of these diseases. Marker-assisted selection (MAS) or Gene-assisted selection (GAS), represent a valuable alternative to the conventional schemes for improving disease resistance using pedigree. Nevertheless a previous knowledge of the genetic factors involved in the trait is necessary to implement this methodology. In this review, the most important aspects of genetic resistance to infectious diseases in salmonids and their suitability for breeding programmes are both reviewed and discussed. Firstly, we briefly mention the most important infectious diseases in Chile. Furthermore, we include aspects related with conventional breeding for this quantitative trait, such as selection criteria, genetic variation of resistance and genetic correlations with other traits. We also review three approaches used in molecular identification of genetic factors involved in resistance: candidate genes, with particular emphasis on the major histocompatibility complex (MHC) or MH genes, detection of quantitative trait loci (QTL) and gene expression studies. Finally, we discuss the use of this information in the implementation of breeding schemes that include disease resistance in their breeding goal.
\end{abstract}

Palabras clave: salmón, resistencia a enfermedades, programas de mejoramiento, QTL.

Key words: salmon, disease resistance, breeding programmes, QTL.

\section{INTRODUCCIÓN}

El cultivo de salmónidos es una de las principales actividades relacionadas con la acuicultura a nivel nacional y una de las que genera productos acuícolas con mayor valor económico a nivel mundial (FAO 2006). Al igual que en otros sistemas intensivos de producción animal el éxito de esta actividad depende en gran medida del control de las enfermedades infecciosas. Un ejemplo claro del impacto que pueden ocasionar ciertas patologías en el cultivo del salmón es la crisis que ha afectado durante los últimos años a la industria salmonicultora nacional como consecuencia de los brotes del virus de la anemia infecciosa del salmón (ISA).

Los programas de mejoramiento genético han permitido aumentar el retorno económico de las explotaciones agropecuarias. El objetivo de mejoramiento debe ser definido para cada especie y para cada población. En general, todas aquellas características de importancia económica deberían estar incluidas en el objetivo de mejoramiento. Por esto, en salmones se incluyen caracteres relacionados con el crecimiento corporal, color y textura de la carne,

\footnotetext{
Aceptado: 23.12.2009.

\# Programa de Becas de Postgrado de CONICYT (21060530)

* Casilla 2 correo 15, La Granja, Santiago, Chile; vmartine@uchile.cl
}

así como también la resistencia genética a enfermedades de tipo viral y bacteriano (Gjedrem 2000). Hasta el momento, los programas de mejoramiento genético han incluido la resistencia a enfermedades solamente en base a la información de parientes, lo cual afecta el grado de progreso genético realizable en cada generación. Lo anterior se debe a que la precisión con la cual se predicen los valores genéticos utilizando información familiar es menor, comparada con la que se obtiene al utilizar la información de los propios candidatos a reproductores (Falconer y Mackay 1996). Además, la selección familiar no permite utilizar la variación del muestreo mendeliano $\mathrm{y}$, al mismo tiempo, es complicado manejar las tasas de consanguinidad a niveles aceptables cuando el número de familias es relativamente bajo (Martínez 2007).

Los marcadores de DNA son variaciones en la secuencia de esta molécula, las que permiten la identificación de genotipos mediante ensayos de laboratorio. Estos marcadores han sido utilizados en la identificación de loci de efecto cuantitativo (QTL) en distintas especies, es decir, en la búsqueda de regiones genómicas que expliquen una proporción relativamente alta de la variación genética para una determinada característica. La información de marcadores moleculares ligados a estos QTL y a genes de efecto mayor puede ser incluida en los programas de mejoramiento genético, en lo que se ha denominado selección asistida por marcadores (MAS) o genes (GAS). 
Estas metodologías pueden ser de gran utilidad en programas que incluyen caracteres complicados de medir en el propio candidato a la selección, como es el caso de la resistencia a enfermedades infecciosas (Meuwissen 2003). Para poder implementar programas de MAS o GAS que incluyan la resistencia a enfermedades dentro del objetivo de mejoramiento, en primer lugar se deben conocer los factores genéticos que explican la variación cuantitativa para esta característica. En los últimos años se ha progresado en la disección del componente genético implicado en la respuesta del hospedero frente a ciertos patógenos, principalmente utilizando modelos animales como pollo y ratón (Qureshi y col 1999). En salmónidos existe reducida información sobre la arquitectura genética de la resistencia a enfermedades, pero es de esperar que con el actual desarrollo y disponibilidad de recursos genómicos en estas especies y el mayor conocimiento sobre la biología de la respuesta inmune en teleósteos, se disponga en el futuro de mayores antecedentes sobre los QTL o genes involucrados en la variación del carácter.

En esta revisión se presentan brevemente los aspectos sanitarios más relevantes para la salmonicultura nacional en la actualidad. Además, se revisan aspectos relacionados con el mejoramiento genético convencional para incrementar la resistencia a enfermedades y las herramientas moleculares utilizadas en la identificación de los factores genéticos involucrados en estos caracteres. Finalmente, se discute sobre la utilidad de la información molecular en la implementación de programas de mejoramiento genético en salmones, que incluyan la resistencia dentro del objetivo de mejoramiento.

\section{ENFERMEDADES INFECCIOSAS DE SALMÓNIDOS EN CHILE}

En Chile el cultivo de salmones ha presentado un desarrollo notable y sostenido durante los últimos veinte años, alcanzando un volumen total de cosecha aproximado de 628 mil toneladas durante el año 2006. ${ }^{1}$ La situación sanitaria de los peces cultivados es uno de los principales factores que afectan el retorno económico de la industria salmonicultora. A pesar del desarrollo científico, profesional y técnico especializado tendiente a mejorar aspectos del manejo sanitario, nuevas condiciones patológicas han ido apareciendo paulatinamente en las distintas especies de peces salmónidos. Desde el punto de vista económico, las enfermedades infecciosas (virales y bacterianas) constituyen las patologías más relevantes (Smith y col 2001).

Dentro de las principales enfermedades bacterianas que afectan al cultivo de peces salmónidos en Chile se pueden mencionar la Piscirickettsiosis o síndrome rickettsial del salmón (SRS), la enfermedad bacteriana del riñón (BKD), el síndrome del alevín de trucha arco-

Salmonchile 2007, http://www.salmonchile.cl/files/T4-Mundial\%20 1996-2006.pdf [consulta: 2-12-2007]. iris (RTFS) y la enfermedad entérica de la boca roja (ERM). Entre las enfermedades virales más relevantes se encuentran la necrosis pancreática infecciosa (IPN), la necrosis eritrocítica viral (VEN) (Smith y col 2001) y recientemente el virus ISA. Una descripción detallada de cada una de estas enfermedades superaría ampliamente el objetivo de esta revisión. Sin embargo, tres de éstas merecen ser destacadas debido a su actual impacto en la salmonicultura nacional. En primer lugar, cabe mencionar que la Piscirickettsiosis ha sido responsable de las mayores pérdidas en la etapa de engorda en la industria chilena del salmón. Por ejemplo, según datos del Instituto Tecnológico del Salmón (INTESAL), institución que monitorea enfermedades entre los productores, en enero del 2007 la Piscirickettsiosis ocasionó más de la mitad $(55,6 \%)$ del total de las mortalidades mensuales en términos de biomasa, tomando en cuenta información obtenida para salmón del Atlántico (Salmo salar), salmón coho (Oncorhynchus kisutch) y trucha arco-iris (Oncorhynchus mykiss) (Leal y Woywood 2007). Por otra parte, a pesar de que en Chile el virus IPN causa menores pérdidas en términos de toneladas de peces muertos, debido a que es una enfermedad que afecta principalmente a alevines, según datos del Fondo de Investigación Pesquera (FIP) los brotes del virus IPN en el país afectaron al $48 \%$ del total de las pisciculturas, al $61 \%$ de los centros de esmoltificación y al $49 \%$ de los centros de engorda en 1999 (FIP 2003), provocando enormes pérdidas al sector. Finalmente, a mediados del 2007 se presentó un brote de ISA ocasionando altas mortalidades en salmón del Atlántico en un centro de cultivo ubicado en Chiloé. A partir de esa fecha se han presentado, consecutivamente, numerosos brotes, lo que ha llevado a considerar al ISA como una enfermedad prevalente en zonas de la X y XI regiones del sur del país, generando grandes pérdidas económicas para la industria (SERNAPESCA 2008).

Considerando que estas enfermedades son de gran relevancia para la industria del salmón en Chile y que las medidas utilizadas para la prevención y el tratamiento de éstas (vacunaciones, antibióticos, etc.) no han mostrado resultados significativos a la fecha, resulta imperativo poder desarrollar nuevas estrategias orientadas a establecer un control efectivo y sustentable de estas patologías. El mejoramiento genético de la resistencia a enfermedades constituye una solución factible al problema (Stear y col 2001). Sin embargo, en el medio nacional la investigación hasta este momento ha sido escasa y no existen trabajos publicados que apunten a determinar los factores genéticos que influencian la resistencia. No obstante lo anterior, en la actualidad se llevan a cabo diversos proyectos de investigación que utilizan herramientas de genética cuantitativa, molecular y genómica funcional para identificar los factores genéticos asociados a la resistencia frente a las tres enfermedades económicamente más relevantes para la industria nacional: Piscirickettsiosis, IPN e ISA. ${ }^{2}$

\footnotetext{
Maldonado, comunicación personal.
} 


\section{MEJORAMIENTO GENÉTICO CONVENCIONAL DE LA RESISTENCIA A ENFERMEDADES INFECCIOSAS}

La resistencia a enfermedades infecciosas se puede definir como la capacidad del hospedero para iniciar y mantener respuestas dirigidas a prevenir el establecimiento de un agente infeccioso y/o eliminarlo del organismo. Seleccionar animales para incrementar su resistencia a enfermedades es un método factible para mejorar la productividad y el bienestar animal, lo que además ofrece ciertas ventajas sobre otros métodos de control frente a las infecciones (Stear y col 2001). En Noruega, desde 1993 se ha incluido la resistencia a enfermedades virales y bacterianas dentro de los programas de mejoramiento convencionales en salmones (Gjøen y Bentsen 1996). Sin embargo, esta metodología presenta algunas dificultades relacionadas principalmente con el criterio de selección utilizado para medir la resistencia. A continuación, se revisan los principales aspectos relacionados con el mejoramiento genético convencional de la resistencia a enfermedades infecciosas en salmónidos.

\section{CRITERIOS DE SELECCIÓN EN EL MEJORAMIENTO CONVENCIONAL DE LA RESISTENCIA}

Existen diferentes criterios de selección que potencialmente podrían ser utilizados para medir la resistencia a enfermedades en programas de mejoramiento en peces. Aunque en algunos esquemas ya se utiliza la selección mediante desafíos experimentales frente a los patógenos, aún permanece abierta la pregunta sobre cuál es el criterio de selección más adecuado, es decir, qué medición debe realizarse en los candidatos para medir la resistencia. A continuación se revisan algunos aspectos estudiados hasta ahora dentro de este ámbito.

Desafío frente a patógenos. La resistencia a enfermedades se puede medir en términos prácticos como la sobrevivencia (y/o mortalidad) de los individuos frente a la infección. Es posible utilizar datos epidemiológicos de brotes de campo para realizar inferencias genéticas sobre la resistencia a enfermedades infecciosas. Para estos efectos, es necesario que el pedigrí de la población pueda ser determinado y que los registros de sobrevivencia se encuentren estructurados como información familiar (Guy y col 2006). Sin embargo, utilizar la información de brotes de campo presenta algunas desventajas como, por ejemplo, la dificultad para reconocer la causa exacta de muerte, debido a que los factores que influencian la sobrevivencia bajo estas condiciones son diversos. Además, la disponibilidad de información depende de la aparición de brotes, los que son generalmente controlados para disminuir las pérdidas económicas. Por otra parte, la inferencia del pedigrí mediante el uso de marcadores moleculares puede incrementar los costos. Sin embargo, los datos de sobrevivencia pueden ser obtenidos desde desafíos experimentales, los cuales pueden ser estandarizados, facilitando la evaluación de los resultados. Asimismo, los desafíos en presmolts son más ventajosos en términos de costos, comparados con los postsmolts utilizados en los estudios de campo. No obstante lo anterior, se requiere que exista una alta correlación entre los resultados obtenidos desde desafíos en pre y postsmolts. A la fecha, se ha reportado una alta correlación genética (hasta un 0,95 ) entre pruebas de campo y desafíos experimentales para furunculosis en salmón del Atlántico (Gjøen y col 1997, Ødegård y col 2006). Por lo tanto, para esquemas de selección que incluyan la resistencia a una determinada enfermedad dentro de su objetivo de mejoramiento, las pruebas de desafío son más precisas y apropiadas que los brotes de campo, ya que disminuyen la variabilidad ocasionada por factores ambientales y resultan más factibles de implementar en la práctica. En efecto, de esta manera es como se determina la resistencia a enfermedades virales y bacterianas en el programa de mejoramiento genético convencional de salmón del Atlántico, llevado a cabo por Aquagen en Noruega (Gjøen y Bentsen 1996, Aquagen 2005).

Variables inmunológicas y fisiológicas. La selección directa para mejorar la resistencia genética a enfermedades, basada en pruebas de desafíos, puede resultar costosa y laboriosa. Además, en programas de mejoramiento convencional sólo puede ser realizada utilizando información de parientes. La selección indirecta, basada en la medición de otras características que se encuentren genéticamente correlacionadas con la resistencia a enfermedades, podría simplificar la recopilación de datos y, al mismo tiempo, podría permitir la incorporación de información individual. Algunos trabajos han apuntado a determinar la variación genética de variables fisiológicas e inmunológicas, y la correlación que existe entre éstos y la sobrevivencia a las pruebas de desafío en salmones. Ejemplos de las variables que han sido estudiadas hasta la fecha son: actividad hemolítica del suero y actividad de lisozimas séricas (Røed y col 1993, Lund y col 1995), niveles de cortisol plasmático (Fevolden y col 1993), IgM y título de anticuerpos (Lund y col 1995), $\alpha 2$-antiplasmina sérica (Salte y col 1993), actividad bactericida y actividad del complemento (Hollebecq y col 1995). Sin embargo, incluso cuando algunos estudios muestran correlaciones significativas entre resistencia y parámetros inmunes, la proporción de la variación total de la sobrevivencia que puede ser explicada por las variables inmunológicas ha sido considerada demasiado baja para ser útil como criterio de selección. Por ello la predicción de valores genéticos para sobrevivencia basándose en estas variables puede no ser adecuada (Gjøen y Bentsen 1996). Esto último resulta razonable considerando la complejidad de los mecanismos implicados en la respuesta inmune y el gran número de factores que pueden estar involucrados en la resistencia a enfermedades, lo que determina una gran dificultad al 
momento de intentar utilizar la información de un solo parámetro en la determinación de la resistencia. Por otro lado, la utilización de todas las variables fisiológicas relacionadas con la sobrevivencia resultaría una metodología cara y menos factible de implementar en la práctica.

\section{VARIACIÓN GENÉTICA DE LA RESISTENCIA A ENFERMEDADES INFECCIOSAS}

Un requisito para mejorar una característica mediante selección artificial es que ésta presente variación genética. La heredabilidad $\left(h^{2}\right)$ expresa la proporción de la variación fenotípica total que es atribuible a la variación genética aditiva (Falconer y Mackay 1996). Es importante tener en cuenta que esta medida no sólo es una propiedad del carácter sino también de la población, de las condiciones ambientales y de la forma en que se evalúa el fenotipo (Falconer y Mackay 1996). Dependiendo de cómo se mida la resistencia a enfermedades, la heredabilidad puede presentar valores distintos e incomparables, debido a diferencias en la definición del carácter y al modelo estadístico utilizado en el análisis (Ødegård y col 2006, Ødegård y col 2007). A la fecha, existe un gran número de trabajos donde se ha descrito variación genética aditiva y se han estimado los valores de heredabilidad para resistencia a diversas enfermedades virales y bacterianas en salmónidos (cuadro 1). Lo anterior, demuestra que el mejoramiento genético de estas características será satisfactorio y constituye una alternativa viable para el control de estas patologías en salmónidos. Sin embargo, se da el caso puntual de la ausencia de estudios de este tipo para la resistencia frente a Piscirickettsiosis, una de las enfermedades más relevantes para la salmonicultura nacional.

\section{CORRELACIÓN GENÉTICA ENTRE RESISTENCIA A ENFERMEDADES INFECCIOSAS Y OTROS CARACTERES DE INTERÉS PRODUCTIVO}

El potencial para mejorar simultáneamente la resistencia genética a distintas enfermedades y otros caracteres económicamente importantes dependerá de las correlaciones genéticas entre estas características. Son pocos los estudios que han apuntado a determinar las correlaciones genéticas para resistencia a enfermedades en salmones. Algunos trabajos en salmón del Atlántico han indicado una correlación genética positiva entre resistencia a distintas enfermedades bacterianas como furunculosis, BKD, vibriosis de agua fría y vibriosis (Gjedrem y Gjøen 1995, Gjøen y col 1997). Sin embargo, existe una correlación genética negativa débil entre la resistencia a la enfermedad viral ISA y distintas enfermedades bacterianas como furunculosis, vibriosis de agua fría y vibriosis (Gjøen y col 1997). En trucha arco-iris se analizó la correlación genética entre la enfermedad septicemia hemorrágica viral (VHS) y las enfermedades bacterianas ERM y RTFS. Al contrario de lo observado en salmón del Atlántico, la correlación

Cuadro 1. Valores de heredabilidad $\left(h^{2}\right)$ y su error estándar $( \pm)$, estimados para resistencia a diferentes enfermedades infecciosas en salmónidos.

Heritability values $\left(h^{2}\right)$ and their standard error $( \pm)$ for resistance to different infectious diseases in salmonids.

\begin{tabular}{|c|c|c|c|}
\hline Enfermedad & Especie & $h^{2} \pm$ & Autores \\
\hline BKD & Salmón coho & $0,53 \pm 0,16$ & Withler y Evelyn (1990) \\
\hline Furunculosis & Salmón del Atlántico & $0,48 \pm 0,17$ & Gjedrem y Col (1991) \\
\hline Vibriosis & Salmón rey & $0,14 \pm 0,11$ & Beacham y Evelyn (1992) \\
\hline Furunculosis & Salmón rey & $0,21 \pm 0,14$ & Beacham y Evelyn (1992) \\
\hline $\mathrm{BKD}$ & Salmón rey & $0 \pm 0,05$ & Beacham y Evelyn (1992) \\
\hline VHS & Trucha arco-iris & $0,63 \pm 0,26$ & Dorson y col (1995) \\
\hline $\mathrm{BKD}$ & Salmón del Atlántico & $0,2 \pm 0,1$ & Gjedrem y Gjøen (1995) \\
\hline Vibriosis de agua fría & Salmón del Atlántico & $0,13 \pm 0,08$ & Gjedrem y Gjøen (1995) \\
\hline Furunculosis & Salmón del Atlántico & $0,34 \pm 0,13$ & Gjøen y col (1997) \\
\hline Vibriosis & Salmón del Atlántico & $0,38 \pm 1,07$ & Gjøen y col (1997) \\
\hline ISA & Salmón del Atlántico & $0,13 \pm 0,03$ & Gjøen y col (1997) \\
\hline Furunculosis & Trucha salmonada & $0,51 \pm 0,03$ & Perry y col (2004) \\
\hline VHS & Trucha arco-iris & $0,11 \pm 0,1$ & Henryon y col (2005) \\
\hline ERM & Trucha arco-iris & $0,21 \pm 0,05$ & Henryon y col (2005) \\
\hline RTFS & Trucha arco-iris & $0,07 \pm 0,02$ & Henryon y col (2005) \\
\hline IPN & Salmón del Atlántico & 0,43 & Guy y col (2006) \\
\hline Furunculosis & Salmón del Atlántico & $0,38 \pm 0,09$ & Ødegård y col (2006) \\
\hline ISA & Salmón del Atlántico & $0,16 \pm 0,01$ & Ødegård y col (2007) \\
\hline
\end{tabular}


genética entre la resistencia a enfermedades bacterianas fue débilmente negativa, mientras que la resistencia a la enfermedad viral estaba favorablemente correlacionada con RTFS y negativamente correlacionada con ERM (Henryon y col 2005). Sin embargo, todas estas correlaciones fueron estimadas con cierto grado de incertidumbre debido al bajo número de familias utilizadas en el estudio (Henryon y col 2005).

Por otra parte, es fundamental conocer la correlación genética que existe entre resistencia a enfermedades y otras características productivas en salmónidos. A la fecha, se han determinado correlaciones genéticas entre resistencia a enfermedades y caracteres relacionados con crecimiento (peso y longitud corporal, tasa de crecimiento y eficiencia de conversión alimenticia). Los resultados varían desde correlaciones genéticas negativas moderadas a bajas (Henryon y col 2002), pasando por correlaciones inconsistentes (Beacham y Evelyn 1992, Henryon y col 2002), hasta correlaciones favorables moderadas y bajas (Standal y Gjerde 1987, Gjedrem y col 1991, Perry y col 2004).

\section{INTERFASE GENÉTICO-MOLECULAR Y MEJORAMIENTO DE LA RESISTENCIA A ENFERMEDADES}

Los grandes avances en la generación y utilización de marcadores moleculares, métodos automatizados de secuenciación y las nuevas técnicas disponibles para el análisis de la información transcriptómica, han contribuido a la identificación QTL y genes asociados a caracteres complejos en diversas especies de vertebrados. Los salmónidos, no se encuentran al margen de este auge en el desarrollo de nuevas técnicas para el análisis genómico. Esto último, se ve reflejado en el considerable incremento de recursos genómicos para especies salmónidas durante los últimos cinco años y en la consolidación de un grupo de colaboración internacional orientado a establecer las prioridades y mantención de estos recursos (Consortium for Genomic Research on All Salmonids Program, cGRASP, Canada). Actualmente, estas herramientas están comenzando a ser utilizadas en la identificación de los factores genéticos involucrados en la resistencia a enfermedades infecciosas en salmónidos. Las estrategias utilizadas en el estudio de la arquitectura genética de la resistencia están basadas principalmente en el análisis de genes candidatos, mapeo de QTL y en estudios de expresión génica.

\section{GENES CANDIDATOS}

La teoría del gen candidato afirma que una proporción significativa de la variación fenotípica de una característica en una población está determinada por la presencia de polimorfismos en genes de efecto mayor involucrados en la expresión de dicho carácter, lo que posibilita la identificación de estos genes (Rothschild y Soller 1997). Esta metodología requiere del conocimiento de la biología de la especie, vías bioquímicas y, principalmente, las secuencias génicas, para poder estudiar la variación en candidatos específicos. En acuicultura, la disponibilidad de secuencias génicas es escasa, pero se espera que un mayor número de genes sea incorporado en las bases de datos públicas en el corto plazo.

En vertebrados, el complejo mayor de histocompatibilidad o MHC ha acaparado una gran atención en estudios de asociación entre variantes génicas y resistencia a enfermedades. Sin embargo, se han propuesto y estudiado otros genes que también pueden jugar un rol importante en el mecanismo de resistencia en especies productivas, organismos modelo y humanos (Hill 1999, Qureshi y col 1999). A la fecha, no existen trabajos que apunten a establecer la asociación que hay entre otros genes candidatos, distintos al MHC, y la resistencia a enfermedades infecciosas en salmones.

Complejo mayor de histocompatibilidad (MHC). El MHC es una familia multigénica que actúa en la interfase entre el sistema inmune y las enfermedades infecciosas. La familia de genes MHC comprende dos subfamilias: Genes clase I y clase II (Bernatchez y Landry 2003). Ambas clases corresponden a glicoproteínas de membrana que participan en el procesamiento y eliminación de patógenos (Thorgaard y col 2002). Los genes MHC clase I son expresados en la superficie de todas las células somáticas nucleadas. Cumplen un importante rol en la defensa inmune contra patógenos intracelulares, ligando péptidos, principalmente virales, en el citoplasma y presentándolos a los linfocitos TCD8+ (Bernatchez y Landry 2003, Grimholt y col 2003).

En vertebrados, existen dos tipos de MHC clase I: Clásico (Ia) y no-clásico (Ib) (Klein y O’Huigin 1994). Los genes MHC clase Ia son altamente polimórficos, expresados en la mayoría de los tejidos y durante la infección su transcripción es modulada por elementos promotores específicos (respuesta mediada por interferón). Por el contrario, los genes MHC clase Ib muestran menos polimorfismo, expresión restringida y no son modulados a nivel transcripcional durante la infección (Thorgaard y col 2002).

Por otro lado, los genes MHC clase II tienen un patrón de expresión más restringido, ya que se expresan en células presentadoras de antígeno (linfocitos B y macrófagos). Básicamente, están involucrados en la mantención del ambiente extracelular presentando antígenos, principalmente bacterianos, a los linfocitos TCD4+ (Bernatchez y Landry 2003, Grimholt y col 2003).

Los genes MHC han sido identificados, clonados y caracterizados en salmón del Atlántico, trucha arco-iris y en otros salmónidos (Grimholt y col 1993, Hordvik y col 1993, Hansen y col 1996, Shum y col 1999, Shum y col 2002). Además, se ha demostrado que estos genes son altamente polimórficos en estas especies (Grimholt y col 1994, Miller y Withler 1996, Hansen y col 1999, Garrigan y Hedrick 2001, Aoyagi y col 2002, Grimholt 
y col 2002). Al igual que en otros vertebrados, existen dos tipos de genes clase I, los UAA que son altamente divergentes, no polimórficos y expresados en bajos niveles, y los UBA que son polimórficos, expresados en altos niveles en bazo y con características estructurales similares a las de las moléculas clase Ia (clásicas), que presentan antígenos a los linfocitos T (Shum y col 1999). Los genes clase II se dividen en: clase II A $(D A A)$ y II B o $(D A B)$, dependiendo si codifican para la cadena $\alpha$ o $\beta$ de la molécula, respectivamente (Grimholt y col 2000, Stet y col 2002). Ambos loci ( $D A A$ y $D A B$ ) cosegregan como haplotipos, lo que sugiere un estrecho ligamiento físico entre ellos en salmón del Atlántico (Stet y col 2002). Lo anterior, sumado a la expresión de un locus único de genes MH clase I (Grimholt y col 2002), podría permitir asociar de manera más sencilla alelos MH y resistencia a enfermedades en esta especie (Stet y col 2002).

En teleósteos, a diferencia de lo que ocurre en otros vertebrados, los genes MH clase I y II segregan de manera independiente (Hansen y col 1996, Bingulac-Popovic y col 1997, Sato y col 2000), por lo tanto, en lugar de hablar de un 'complejo' mayor de histocompatibilidad (MHC), es más apropiado hablar de 'genes MH' en estas especies (Grimholt y col 2003). La ausencia de ligamiento entre los genes clase I y clase II tiene implicancias potenciales en la selección natural de los polimorfismos en ambas clases. $\mathrm{Si}$ estos dos tipos de genes estuviesen ligados, la selección de un determinado alelo clase II inevitablemente cambiaría la frecuencia de un alelo ligado clase I y viceversa (Shum y col 2001). Además, la ausencia de ligamiento permite una segregación independiente de los caracteres inmunológicos en peces, asociados a los genes clase I (citotoxicidad en respuesta a la infección viral) y genes clase II (respuesta humoral frente a bacterias).

Se ha analizado la asociación entre un polimorfismo ligado a genes $\mathrm{MH}$ clase II y la resistencia frente al virus de la necrosis hematopoiética infecciosa (IHN), en retrocruzas de trucha arco-iris y trucha cutthroat (Oncorhynchus clarki). El efecto del polimorfismo en la sobrevivencia fue muy pequeño y significativo en una de las dos familias analizadas, lo que sugiere que esta mutación puede estar ligada a un gen que influencia la resistencia a IHN. Sin embargo, los efectos ambientales y la participación de otros genes involucrados en la característica pueden atenuar el efecto de este locus (Palti y col 2001). En salmón del Atlántico se ha demostrado la asociación entre alelos MH clase IIB específicos y la resistencia frente Aeromonas salmonicida (Langefors y col 2001, Lohm y col 2002). En esta misma especie se han asociado variantes de genes MH clase I y clase II, con susceptibilidad frente a IHN (Miller y col 2004). Por otra parte, se ha determinado la asociación entre alelos específicos de genes MH clase I y II y la resistencia frente a ISA y furunculosis, de manera independiente (Grimholt y col 2003). Basándose en los resultados de este trabajo, se seleccionaron genotipos resistentes y susceptibles, los que posteriormente fueron cruzados para generar una progenie con genotipos para alta y baja mortalidad esperada. El estudio confirmó parcialmente las expectativas en cuanto a resistencia y susceptibilidad de alelos individuales MH clase I y IIA frente a ISA, establecidas anteriormente por Grimholt y col (2003). Ello debido a que algunas variantes que mostraban estar asociadas a resistencia en el estudio anterior no establecieron un aumento de la sobrevivencia en este segundo ensayo; sin embargo, las variantes asociadas previamente con susceptibilidad repitieron su desempeño (Kjøglum y col 2006).

A pesar de las asociaciones que se han establecido entre variantes de genes $\mathrm{MH}$ y resistencia a enfermedades, se ha demostrado que no es el único factor genético que influencia la variación de esta característica en salmones. En salmón del Atlántico se ha detectado que existe un efecto que no está relacionado con los genes $\mathrm{MH}$, en cuanto a la resistencia frente a IPN, furunculosis e ISA (Kjøglum y col 2005). Este efecto, cercano al $10 \%$ en promedio, para las tres enfermedades produce variación genética significativa en la sobrevivencia de las familias desafiadas (idénticas en alelos MH), lo que se puede explicar sobre la base de una diferencia en los efectos poligénicos asociados a la característica. Además, en este estudio se detectó un efecto asociado al tanque, el que influencia de manera significativa la variación observada para la resistencia frente a IPN. Por lo tanto, este factor técnico debe ser considerado al momento de analizar la resistencia genética a enfermedades infecciosas, mediante pruebas de desafío (Kjøglum y col 2005). Debido a la naturaleza poligénica de la resistencia a enfermedades, es importante considerar el trasfondo genómico y las interacciones que pueden ocurrir entre genes (epístasis), lo que dificulta aún más el análisis de genes candidatos individuales involucrados en la variación de la resistencia.

\section{DETECCIÓN DE QTL}

El mapeo de QTL es una estrategia que puede entregar información sobre la ubicación y el efecto de los genes que se encuentran influenciando una característica cuantitativa compleja, tal como la resistencia a enfermedades infecciosas. Las metodologías de detección de QTL se basan en la utilización de marcadores de DNA para identificar regiones genómicas involucradas en la variación genética de un determinado carácter de interés productivo.

Marcadores de DNA. El desarrollo de marcadores basados en variaciones del DNA ha generado un gran impacto en los estudios de la variación genética en animales y peces. Dentro de los marcadores de DNA más ampliamente utilizados se encuentran los RFLPs (Polimorfismos de longitud de fragmentos de restricción), RAPDs (Amplificación aleatoria de DNA polimórfico), AFLPs (Amplificación de fragmentos de longitud polimórfica), microsatélites y SNPs (Polimorfismos de nucleótido único). Estos varían 
entre sí en su modo de herencia, métodos de identificación y detección, cantidad de locus que abarcan y contenido de información polimórfica (Liu y Cordes 2004).

Los AFLPs presentan la ventaja de que pueden ser generados más fácilmente y a menor costo que SNPs y microsatélites, debido a que no es necesario contar con información genómica para su generación. Sin embargo, su modo de herencia, al igual que los RAPDs es codominante, es decir, no es posible diferenciar genotipos heterocigotos de homocigotos dominantes sin la utilización de equipos y software especiales (Piepho y Koch 2000). Lo anterior reduce la cantidad de información entregada por este tipo de marcadores.

Los microsatélites son secuencias nucleotídicas, entre 1 a 6 pares de bases, repetidas en tándem. Los diferentes alelos se generan debido a la variación en el número de repeticiones. Entre sus características principales se encuentran el ser altamente variables, presentar herencia codominante y ser abundantes y encontrarse ampliamente distribuidos en el genoma. La gran ventaja de los microsatélites es su alto grado de polimorfismo. Además, su análisis está basado en la amplificación del DNA mediante la reacción en cadena de la polimerasa (PCR), de modo que se realiza de forma rápida, a bajo costo y la cantidad de DNA necesario es mínima (del orden de nanogramos). En salmónidos existe un gran número de microsatélites disponibles para su utilización en estudios genéticos (Phillips y col 2009).

Por otra parte, los SNPs pueden estar presente tanto en regiones codificantes como no codificantes. Generalmente, son bialélicos, se distribuyen uniformemente en el genoma y son más abundantes que los microsatélites. Por lo tanto, son ideales para la construcción de mapas genéticos densos, los cuales pueden ser utilizados en el mapeo fino de QTL y facilitar la identificación de genes causales de la variación genética para determinados caracteres. Recientemente, basándose en el alineamiento de más de 100.000 secuencias de ESTs (Marcadores de secuencias expresadas) se han detectado más de 2.500 SNPs putativos en Salmo salar, con una tasa de validación mayor a un $70 \%$ (Hayes y col 2007).

Detección de QTL para resistencia a enfermedades en salmónidos. El desarrollo de un mapa genético es el primer paso hacia la identificación de QTL. A la fecha, se han construido mapas de ligamiento para trucha arco-iris (Young y col 1998, Sakamoto y col 2000, Nichols y col 2003, Guyomard y col 2006), salmón del Atlántico (Moen y col 2004 ${ }^{\mathrm{b}}$, Gilbey y col 2004), trucha común (Salmo trutta) (Gharbi y col 2006) y trucha ártica (Salvelinus alpinus) (Woram y col 2004).

Estudios con microsatélites orientados a detectar QTL para resistencia a enfermedades en salmónidos, han identificado dos QTL de mediano y gran efecto para resistencia frente a IPN en trucha arco-iris. Estos loci explicaron una gran proporción (27\% y $34 \%$ ) de la variación fenotípica en una familia proveniente de una retrocruza de una cepa susceptible a IPN (YK-RT101) y otra resistente (YN-RT201) (Ozaki y col 2001).

En esta misma especie, utilizando AFLPs y microsatélites, se han detectado marcadores asociados con resistencia a IHN dentro de tres grupos de ligamiento diferentes (Rodríguez y col 2004). Para esta misma enfermedad se han detectado marcadores RFLPs asociados a familias resistentes y susceptibles, en retrocruzas de trucha arcoiris y trucha cutthroat (Palti y col 1999).

En salmón del Atlántico, con una estrategia múltiple que combina el Test de Transmisión de Desequilibrio (TDT), la determinación de la segregación mendeliana de los marcadores y análisis de sobrevivencia, se logró detectar dos QTL asociados a resistencia frente a ISA, mediante el uso de marcadores AFLPs en dos familias de propios hermanos (Moen y col 2004 ${ }^{\mathrm{a}}$ ). En un estudio más reciente, se ha logrado validar la presencia de uno de los QTL detectados por Moen y col (2004) ), esta vez utilizando marcadores tipo microsatélites en un mayor número de peces. Este QTL explicó el 6\% de la variación fenotípica para la resistencia frente a ISA y se ha localizado en el grupo de ligamiento 8 del salmón del Atlántico (siguiendo la notación SALMAP) (Moen y col 2007).

En esta misma especie utilizando las tasas de recombinación diferenciales entre sexos se han detectado tres QTL para resistencia frente a IPN mediante una metodología basada en dos etapas. En primer lugar, se realizó un análisis utilizando sólo las recombinaciones de los machos para determinar los grupos de ligamiento con efectos significativos, empleando entre dos a tres microsatélites por cromosoma. Posteriormente, usando las recombinaciones de las hembras y un mayor número de marcadores por grupo de ligamiento se confirmaron y posicionaron los QTL previamente detectados (Houston y col 2008 ). El QTL más significativo, mapeado en el grupo de ligamiento 21 , fue posteriormente confirmado mediante el análisis de nueve familias adicionales y una mayor saturación de marcadores (Houston y col 2008 ${ }^{\mathrm{a}}$ ).

En general los intervalos de confianza para los QTL detectados son extensos. Esto tiene dos consecuencias. En primer lugar, intervalos amplios pueden contener un gran número de genes (miles) y, por lo tanto, identificar el polimorfismo causal de la variación resulta muy complejo. En segundo lugar, el uso de estos QTL en programas de MAS es complicado, ya que la fase de ligamiento entre el marcador y el QTL a través de toda la población puede ser distinta a la determinada en las familias sometidas al estudio. Una alternativa para reducir los intervalos de confianza en el mapeo de QTL es utilizar la información proveniente del análisis de ligamiento en conjunto con la información del LD poblacional (Meuwissen y col 2002). Mediante estudios de simulación, metodologías que utilizan esta información conjunta han sido probadas con éxito en el mapeo fino de QTL en poblaciones comerciales de salmones (Hayes y col 2006). Lo anterior, en conjunto con 
la disponibilidad de mapas genéticos densos, contribuirán a disminuir los intervalos de confianza en la identificación de QTL en estas especies. La información de estos QTL, junto con la secuenciación y el mapeo físico del genoma, facilitarán la determinación de las mutaciones involucradas en la resistencia, a través de estudios posicionales. Estas mutaciones podrán ser utilizadas directamente en programas de selección genética.

\section{GENÓMICA FUNCIONAL}

La genómica funcional, definida como la aplicación de métodos experimentales de amplia cobertura genómica o sistémica para evaluar la función génica a partir de datos y antecedentes procedentes de la genómica estructural (mapeo y secuenciación), se está convirtiendo en un área de interés primordial (Hiendleder y col 2005). La idea básica de las metodologías utilizadas en esta área es ampliar el espectro de la investigación biológica a un nivel holístico donde se estudien, de manera simultánea, gran cantidad de genes transcritos y proteínas. Actualmente, los recursos genómicos disponibles en salmónidos han permitido algunas aproximaciones al estudio de la respuesta frente a enfermedades infecciosas utilizando herramientas de genómica funcional.

Expresión génica. Genes cuyos niveles de expresión se modifican en respuesta a una infección pueden ser identificados mediante técnicas de alto rendimiento (High-throughput). Por ejemplo, mediante una técnica denominada hibridación sustractiva bajo condiciones de supresión (SSH) y utilizando muestras de hígado de individuos inyectados con una bacterina de $V$. anguillarum e individuos normales, se han detectado más de 25 genes importantes en la respuesta inmune en trucha arco-iris, incluyendo secuencias de proteínas de fase aguda de la inflamación, sistema del complemento y coagulación (Bayne y col 2001). Mediante la misma técnica, genes involucrados en transducción de señales, inmunidad innata y otros procesos han sido identificados como relevantes frente al desafío con A. salmonicida en salmón del Atlántico (Tsoi y col 2004).

Por otro lado, la disponibilidad de librerías de ESTs y cDNAs ha permitido el desarrollo de microarreglos de DNA que pueden ser utilizados para el estudio de la expresión diferencial de un gran número de genes de manera simultánea en salmónidos (Rise y col 2004b, Ewart y col 2005, von Schalburg y col 2005). Utilizando un microarreglo de cDNAs humanos se han identificado transcritos de salmón del Atlántico expresados diferencialmente frente al desafío con A. salmonicida. Sin embargo, debido a la divergencia de las especies, sólo el $6 \%$ de las secuencias del microarreglo mostraron hibridación detectable contra el cDNA de hígado de salmón (Tsoi y col 2003). Rise y $\operatorname{col}\left(2004^{\mathrm{a}}\right)$ llevaron a cabo el primer estudio basado en un microarreglo construido a partir de librerías de cDNAs de salmones. De esta manera, en salmón del Atlántico se han identificado genes que presentan expresión diferencial, dependiendo si provienen de macrófagos infectados o no infectados con Piscirickettsia salmonis, y riñón hematopoyético de individuos desafiados o no desafiados frente al mismo patógeno. Estos genes, caracterizados por anotaciones funcionales de relevancia inmune, podrían ser utilizados como biomarcadores moleculares de la infección con P. salmonis (Rise y col 2004ª). Además, utilizando un microarreglo de cDNA de salmón del Atlántico con más de 4.000 genes extraídos desde hígado, bazo y riñón anterior, se han descubierto varios genes diferencialmente expresados en la respuesta frente a la infección por $A$. salmonicida (Ewart y col 2005). Por otra parte, se ha analizado la respuesta transcriptómica frente a la vacunación con una bacterina de A. salmonicida en salmón del Atlántico, revelando diferencias temporales y tisulares en cuanto a los niveles de expresión, lo que puede tener relevancia en el establecimiento de la protección (Martin y col 2006). Por otra parte, se ha estudiado el perfil de expresión génica en respuesta a una vacuna de DNA del virus IHN en trucha arco-iris, detectando 910 genes modulados en el sitio de la inyección y, además, se determinó la sobreexpresión de genes relacionados con el sistema interferón tipo I (IFN-1) en otros tejidos, sugiriendo que este sistema es la base de la inmunidad antiviral temprana (Purcell y col 2006). En los estudios anteriormente mencionados, ciertos transcritos que mostraban variación en los niveles de expresión frente a la infección no presentaron homólogos en GenBank, por lo que sus funciones permanecen siendo desconocidas (Rise y col 2004', Ewart y col 2005, Martin y col 2006, Purcell y col 2006). Por lo tanto, se debe trabajar basándose en las secuencias de las proteínas codificadas y en su rol en la respuesta inmune para descifrar su función frente a la infección.

A la fecha no existen estudios orientados a analizar la expresión diferencial entre peces resistentes y susceptibles para una determinada infección. La información entregada por este tipo de análisis puede ser utilizada en el descubrimiento de nuevos grupos de genes, con o sin una función asignada, que podrían estar relacionados con la resistencia a enfermedades (Walsh y Henderson 2004). Sin embargo, se debe tener en cuenta que genes expresados diferencialmente pueden estar, probablemente, actuando en trans, es decir, cuya expresión está regulada por otros genes (Martínez 2007), lo que va a tener implicancias al momento de asociar polimorfismos de éstos con la resistencia. Otra posibilidad es considerar los niveles de expresión diferencial como un carácter cuantitativo. Esto podría permitir la identificación de QTL asociados a diferencias en los patrones de expresión génica, entre individuos resistentes y susceptibles (eQTL) (Pomp y col 2004, de Koning y col 2005). Sin embargo, aún es poco claro cómo la información entregada por análisis de expresión puede ser utilizada en programas de mejoramiento genético (Walsh y Henderson 2004). 
SELECCIÓN ASISTIDA POR MARCADORES MOLECULARES (MAS) Y SELECCIÓN ASISTIDA POR GENES (GAS)

Un parámetro crucial en la detección de QTL y en la posterior aplicación de programas de MAS es el nivel de desequilibrio de ligamiento (LD) que existe entre los marcadores y las mutaciones causales de la variación del carácter a nivel poblacional.

\section{DESEQUILIBRIO DE LIGAMIENTO}

Considerando 2 loci, A y B, cada uno con dos alelos, $\mathrm{A} / \mathrm{a}$ y $\mathrm{B} / \mathrm{b}$, el $\mathrm{LD}$ se define como: $\mathrm{P}(\mathrm{A} \mid \mathrm{B}) \neq \mathrm{P}(\mathrm{Alb})$, donde $\mathrm{P}(\mathrm{A} \mid \mathrm{B})$ representa la frecuencia de $\mathrm{A}$ entre los gametos que contienen B y $\mathrm{P}(\mathrm{Alb})$ la frecuencia de A entre los gametos que contienen $b$ (Goddard y Meuwissen 2005). Dicho de otra manera, la probabilidad de A dado B es distinta a la probabilidad de A dado b. El equilibrio de ligamiento (LE), por lo tanto, es la situación contraria: $\mathrm{P}(\mathrm{A} \mid \mathrm{B})=\mathrm{P}(\mathrm{Alb})$. Entre los factores que gobiernan el LD en una población se encuentran las mutaciones, la selección, la deriva (consanguinidad) y la migración o cruzamientos. Uno de los factores que disminuye el nivel de LD en cada generación es la recombinación. Los programas de MAS pueden ser implementados a través de poblaciones en LE o LD entre marcadores y QTL, o basados en la selección directa de la mutación que se encuentra explicando el efecto del QTL (GAS).

\section{MAS EN POBLACIONES EN LE}

Cuando LD entre el QTL y el marcador sólo existe dentro de familias y no a través de toda la población, las tasas de recombinación pueden romper la asociación existente entre alelos del marcador y del QTL entre familias. Por lo tanto, la fase de ligamiento entre el marcador y el QTL debe ser determinada en cada generación y para cada familia por separado. Para determinar si el marcador y el QTL se encuentran en LD dentro de cada familia se necesitan registros fenotípicos y genotipos en cada generación. Esto hace que la implementación de MAS utilizando marcadores en LD con el QTL sólo dentro de familias sea poco atractiva (Dekkers y van der Werf 2007). En el caso de la resistencia a enfermedades, todas las familias disponibles dentro del programa deben ser desafiadas en cada generación, como ocurre en los esquemas convencionales.

\section{MAS EN POBLACIONES EN LD}

Utilizando la información de mapas densos es posible hacer uso del LD entre marcadores y la mutación causal de la variación del carácter a través de toda la población (LD entre familias). Existen dos posibilidades para explotar el LD poblacional en esquemas de MAS. Se puede utilizar la información del efecto de un determinado haplotipo en $\mathrm{LD}$ con el polimorfismo beneficioso a través de la población o se puede predecir el valor genético de un individuo a partir de mapas densos de amplia cobertura genómica (Selección genómica) (Lande y Thompson 1990, Meuwissen y col 2001). La efectividad de estas estrategias depende de la magnitud de los efectos asociados con el o los polimorfismos. Cuando se estiman los efectos de los haplotipos a través del genoma completo, es posible utilizar estos efectos para seleccionar en generaciones posteriores a la estimación inicial, sin necesidad de contar con los fenotipos (Meuwissen y col 2001). La recombinación hará decaer el LD en cada generación y la magnitud de esta disminución dependerá de varios parámetros poblacionales (Meuwissen y col 2001). En la práctica, es necesario verificar la respuesta a la selección en cada generación y para esto se pueden reestimar los efectos utilizando una muestra aleatoria de individuos de la población.

GAS

Se ha determinado que al utilizar GAS en poblaciones en LE se obtiene un mayor progreso genético en relación al alcanzable al utilizar MAS. Esto se debe a que en esquemas de MAS se seleccionan marcadores asociados al QTL y, en el caso de GAS, se selecciona directamente el polimorfismo favorable (Villanueva y col 2002). Sin embargo, en la práctica es probable que los programas de MAS se lleven a cabo utilizando la información de un número importante de marcadores para predecir los efectos alélicos de más de un QTL simultáneamente, mientras en programas de GAS, probablemente sólo un limitado número de polimorfismos estarán disponibles. Por lo tanto, esquemas de MAS pueden generar mayor progreso genético, debido a que utilizan una mayor proporción de la varianza genética. Sin embargo, es necesario tener en cuenta el costo asociado a genotipar una mayor cantidad de marcadores (Martínez 2007).

Entre los factores que influyen en la rentabilidad de GAS se incluyen: la cantidad de variación explicada por los genes disponibles; la frecuencia de los alelos favorables y la disponibilidad de un test para la población en estudio, los posibles efectos pleiotrópicos y los costos asociados a la implementación del genotipado. Por lo tanto, es necesario evaluar estos factores desde un punto de vista económico y determinar su beneficio en comparación con las otras metodologías descritas.

\section{UTILIZACIÓN DE MAS EN EL MEJORAMIENTO} DE LA RESISTENCIA

El mejor predictor lineal insesgado (BLUP) utiliza la información conjunta del pedigrí y fenotipos para predecir los valores genéticos de los individuos. Los marcadores moleculares entregan una nueva fuente de información cuyo mayor impacto estará dado en la precisión al momento de predecir los valores genéticos. De esta forma, la respuesta a la selección será sustancialmente mejor en caracteres 
en los cuales la precisión es baja, es decir, caracteres con bajas heredabilidades o caracteres que no se pueden medir en los propios candidatos a la selección, como es el caso de la resistencia a enfermedades (Meuwissen 2003). El incremento relativo en la precisión depende de la cantidad de variación explicada por los marcadores, la cual depende del número de QTL identificados y utilizados en los esquemas de selección (Lande y Thompson 1990).

En especies productivas se ha demostrado que los efectos de los QTL presentan una distribución leptocúrtica, con un pequeño número de loci de gran efecto y un mayor número de loci de efecto pequeño (Hayes y Goddard 2001), lo cual probablemente es el caso en especies acuícolas (Martínez y col 2005). Por lo tanto, es de esperar que más de un solo marcador sea necesario para que los programas de MAS sean eficientes (Martínez 2007). Además, se deben considerar los efectos pleiotrópicos de los distintos polimorfismos y los posibles efectos negativos en otras características. Por ejemplo, las correlaciones genéticas negativas detectadas entre resistencia a enfermedades virales y bacterianas pueden constituir un problema si el objetivo es seleccionar para resistencia frente a un amplio rango de patógenos. Por otra parte, el no considerar los efectos genéticos no aditivos en el modelo de análisis podría disminuir la precisión con la cual se estiman los efectos genético-aditivos.

Es probable que en el futuro el genotipado de SNPs a gran escala permita la utilización de estos marcadores en la selección de reproductores de una manera costo-efectiva. Por lo tanto, se puede esperar que programas de MAS utilizando marcadores en LD a través de la población sean implementados a nivel de genoma completo. Sin embargo, es necesario determinar el beneficio económico de la utilización de un solo haplotipo versus la utilización de múltiples haplotipos y establecer cuál de los dos métodos se ajusta mejor a la población bajo selección.

Asimismo, en la práctica existen otros factores que deberían ser considerados al momento de utilizar la información de QTL para el mejoramiento de la resistencia a enfermedades en salmónidos como, por ejemplo, las características epidemiológicas de las distintas patologías y las consideraciones económicas relativas a la implementación del programa.

\section{CONCLUSIONES}

Existe reducida y dispersa información relacionada con los factores genéticos involucrados en la resistencia a enfermedades infecciosas en salmones. Sin embargo, el incipiente desarrollo de recursos genómicos en estas especies entregará nuevas herramientas para la disección genética de estas características. La utilización de estas herramientas resultará de gran ayuda para identificar loci o genes que influencian de manera significativa la variación de estos caracteres. Esta información será fundamental para poder implementar programas de MAS o GAS que incluyan la resistencia dentro del objetivo de mejoramiento. Estas metodologías incrementarán la precisión en la selección de los candidatos a reproductores, mejorando de esta forma, la respuesta a la selección. Sin embargo, se debe estudiar para cada caso la factibilidad económica en la implementación de estas nuevas estrategias y el beneficio obtenido en comparación con los esquemas de selección convencionales.

\section{RESUMEN}

El control de las enfermedades infecciosas es fundamental en el éxito del cultivo del salmón. El mejoramiento genético de la resistencia a enfermedades puede otorgar una opción factible y sustentable para el control de éstas. La Selección Asistida por Marcadores Moleculares (MAS) o Genes (GAS) se proyecta como una valiosa alternativa al mejoramiento convencional de la resistencia. Sin embargo, para implementar esta metodología es necesario el conocimiento previo de los factores genéticos involucrados en el carácter. En este trabajo se revisan y se discuten los aspectos más relevantes de la resistencia genética a enfermedades infecciosas en salmónidos y su aplicabilidad a programas de mejoramiento. En primer lugar, se presentan brevemente las enfermedades infecciosas más relevantes a nivel nacional. Además, se incluyen aspectos relacionados con el mejoramiento convencional para este rasgo cuantitativo, tales como criterios de selección, variación genética de la resistencia y correlaciones genéticas con otros caracteres de interés productivo. Por otra parte, se revisan tres aproximaciones moleculares utilizadas en la identificación de los factores genéticos involucrados en la resistencia: genes candidatos, con especial énfasis en el complejo mayor de histocompatibilidad (MHC) o genes MH, detección de loci de efecto cuantitativo (QTL) y estudios de expresión génica. Finalmente, se revisa y se discute en relación a la utilización de esta información molecular en la implementación de programas de mejoramiento genético que incluyan la resistencia a enfermedades infecciosas dentro de su objetivo de selección.

\section{REFERENCIAS}

Aoyagi K, JM Dijkstra, C Xia, I Denda, M Ototake, K Hashimoto, T Nakanishi. 2002. Classical MHC class I genes composed of highly divergent sequence lineages share a single locus in rainbow trout (Oncorhynchus mykiss). J Immunol 168, 260-273.

Aquagen. 2005. Selection for increased disease resistance gives results. Information letter $N^{\circ} 5$. Norway.

Bayne CJ, L Gerwick, K Fujiki, M Nakao, T Yano. 2001. Immune relevant (including acute phase) genes identified in the livers of rainbow trout, Oncorhynchus mykiss, by means of suppression subtractive hybridization. Dev Comp Immunol 25, 205-217.

Beacham TD, TPT Evelyn. 1992. Genetic variation in disease resistance and growth of chinook, coho, and chum salmon with respect to vibriosis, furunculosis, and bacterial kidney disease. Trans Am Fish Soc 121, 456-485.

Bernatchez L, C Landry. 2003. MHC studies in nonmodel vertebrates: what have we learned about natural selection in 15 years? J Evol Biol $16,363-377$.

Bingulac-Popovic J, F Figueroa, A Sato, WS Talbot, SL Johnson, M Gates, JH Postlethwait, J Klein. 1997. Mapping of mhc class I and class II regions to different linkage groups in the zebrafish, Danio rerio. Immunogenetics 46, 129-134.

De Koning DJ, Ö Carlborg, C Haley. 2005. The genetic dissection of immune response using gene-expression studies and genome mapping. Vet Immunol Immunopathol 105, 343-352.

Dekkers J, J Van der Werf. 2007. Strategies, limitations and opportunities for marker-assisted selection in livestock. In: Marker-Assisted 
selection - Current status and future perspectives in crops, livestock, forestry and fish. FAO, Rome, Italy, Pp 167-184.

Dorson M, E Quillet, MG Hollebecq, C Torhy, B Chevassus. 1995. Selection of rainbow trout resistance to viral haemorrhagic septicaemia virus and transmission of resistance by gynogenesis. Vet Res 26, 361-368.

Ewart K, J Belanger, J Williams, T Karakach, S Penny, S Tsoi, R Richards, $S$ Douglas. 2005. Identification of genes differentially expressed in Atlantic salmon (S. salar) in response to infection by Aeromonas salmonicida using cDNA microarray technology. Dev Comp Immunol 29, 333-347.

Falconer DS, TFC Mackay. 1996. Introduction to Quantitative Genetics. $4^{\text {th }}$ ed. Essex, UK

FAO, Food and Agriculture Organization of the United Nations. 2006. State of world aquaculture. FAO Fisheries technical paper. No. 500. Rome, Italy.

Fevolden SE, R Nordmo, T Refstie, KH Røed. 1993. Disease resistance in Atlantic salmon (Salmo salar) selected for high or low response to stress. Aquaculture 109, 215-224.

FIP, Fondo de Investigación Pesquera, Chile. 2003. Riesgos de introducción de enfermedades infectocontagiosas en salmónidos. Informe final Proyecto FIP 2001-08.

Garrigan D, PW Hedrick. 2001. Class I MHC polymorphism and evolution in endangered California Chinook and other Pacific salmon. Immunogenetics 53, 483-489.

Gharbi K, A Gautier, RG Danzmann, S Gharbi, T Sakamoto, B Hoyheim, JB Taggart, M Cairney, R Powell, F Kreig, N Okamoto, MM Ferguson, LE Holm, R Guyomard. 2006. A linkage map for brown trout (Salmo trutta): chromosome homeologies and comparative genome organization with other salmonid fish. Genetics 172, 2405-2419.

Gilbey J, E Verspoor, A McLay, D Houlihan. 2004. A microsatellite linkage map for Atlantic salmon (Salmo salar). Anim Genet 35, 98-105.

Gjedrem T, R Salte, HM Gjøen. 1991. Genetic variation in susceptibility of Atlantic salmon to furunculosis. Aquaculture 97, 1-6.

Gjedrem T, HM Gjøen. 1995. Genetic variation in susceptibility of Atlantic salmon (Salmo salar L.) to furunculosis, BKD, and coldwater vibriosis. Aquac Res 26, 129-134.

Gjedrem T. 2000. Genetic improvement of cold-water fish species. Aquac Res 31, 25-33.

Gjøen HM, HB Bentsen. 1997. Past, present and future of genetic improvement in salmon aquaculture. ICES J Mar Sci 54, 1009-10014.

Gjøen HM, T Refstie, O Ulla, B Gjerde. 1997. Genetic correlations between survival of Atlantic salmon in challenge and field tests. Aquaculture 158, 277-288.

Goddard, ME, THE Meuwissen. 2005. The use of linkage disequilibrium to map quantitative trait loci. Aust J Exp Agr 45, 837-845.

Grimholt U, I Hordvik, VM Fosse, I Olsaker, C Endresen, O Lie. 1993. Molecular cloning of major histocompatibility complex class I cDNAs from Atlantic salmon, Salmo salar L. Immunogenetics 37, 469-473.

Grimholt U, I Olsaker, C de Vries Lindstrom, O Lie. 1994. A study of variability in the MHC class II beta- 1 and class I alpha-2 domain exons of Atlantic salmon, Salmo salar L. Anim Genet 25, 147-153.

Grimholt U, A Getahun, T Hermsen, RJ Stet. 2000. The major histocompatibility class II alpha chain in salmonid fishes. Dev Comp Immunol 24, 751-763.

Grimholt U, F Drablos, SM Jorgensen, B Hoyheim, RJ Stet. 2002. The major histocompatibility class I locus in Atlantic salmon (Salmo salar $L$.): polymorphism, linkage analysis and protein modelling. Immunogenetics 54, 570-581.

Grimholt U, S Larsen, R Nordmo, P Midtlyng, S Kjoeglum, A Storset, S Saebø, RJ Stet. 2003. MHC polymorphism and disease resistance in Atlantic salmon (Salmo salar); facing pathogens with single expressed major histocompatibility class I and class II loci. Immunogenetics 55, 210-219.

Guy DR, SC Bishop, S Brotherstone, A Hamilton, RJ Roberts, BJ McAndrew, JA Woolliams. 2006. Analysis of the incidence of infectious pancreatic necrosis mortality in pedigreed Atlantic salmon, Salmo salar L., populations. J Fish Dis 29, 637-647.

Guyomard R, S Mauger, K Tabet-Canale, S Martineau, C Genet, F Krieg, E Quillet. 2006. A type I and type II microsatellite linkage map of rainbow trout (Oncorhynchus mykiss) with presumptive coverage of all chromosome arms. BMC Genomics 7, 302.

Hansen JD, P Strassburger, L Du Pasquier. 1996. Conservation of an alpha 2 domain within the teleostean world, MHC class I from the rainbow trout Oncorhynchus mykiss. Dev Comp Immunol 20, 417-425.

Hansen JD, P Strassburger, GH Thorgaard, WP Young, L Du Pasquier. 1999. Expression, linkage, and polymorphism of MHC-related genes in rainbow trout, Oncorhynchus mykiss. J Immunol 163, 774-786.

Hayes B, ME Goddard. 2001. The distribution of the effects of genes affecting quantitative traits in livestock. Genet Sel Evol 33, 209-229.

Hayes B, A Gjuvsland, S Omholt. 2006. Power of QTL mapping experiments in commercial Atlantic salmon populations, exploiting linkage and linkage disequilibrium and effect of limited recombination in males. Heredity 97, 19-26.

Hayes B, JK Laerdahl, S Lien, T Moen, P Berg, K Hindar, WS Davidson, BF Koop, A Adzhubei, B Høyheim. 2007. An extensive resource of single nucleotide polymorphism markers associated with Atlantic salmon (Salmo salar) expressed sequences. Aquaculture 265, 82-90.

Henryon M, A Jokumsen, P Berg, I Lund, PB Pedersen, NJ Olesen, WJ Slierendrecht. 2002. Genetic variation for growth rate, feed conversion efficiency, and disease resistance exists within a farmed population of rainbow trout. Aquaculture 209, 59-76. Erratum: Aquaculture 216, 387-388.

Henryon M, P Berg, NJ Olesen, TE Kjær, WJ Slierendrecht, A Jokumsen, I Lund. 2005. Selective breeding provides an approach to increase resistance of rainbow trout (Onchorhynchus mykiss) to the diseases, enteric redmouth disease, rainbow trout fry syndrome, and viral heamorrhagic septicaemia. Aquaculture 250, 621-636.

Hiendleder S, S Bauersachs, A Boulesteix, H Blum, GJ Arnold, T Fröhlich, E Wolf. 2005. Functional genomics: tools for improving farm animal health and welfare. Rev Sci Tech Off Int Epiz 24, 354-377.

Hill AV. 1999. Genetics and genomics of infectious disease susceptibility. Br Med Bull 55, 401-213.

Hollebecq MG, B Faivre, C Bourmaud, C Michel. 1995. Spontaneous bactericidal and complement activities in serum of rainbow trout (Oncorhynchus mykiss) genetically selected for resistance or susceptibility to furunculosis. Fish Shellfish Immunol 5, 407-426.

Hordvik I, U Grimholt, VM Fosse, O Lie, C Endresen. 1993. Cloning and sequence analysis of cDNAs encoding the MHC class II beta chain in Atlantic salmon Salmo salar. Immunogenetics 37, 437-441.

Houston RD, A Gheyas, A Hamilton, DR Guy, AE Tinch, JB Taggart, BJ McAndrew, CS Haley, SC Bishop. 2008 a Detection and confirmation of a major QTL affecting resistance to infectious pancreatic necrosis (IPN) in Atlantic salmon (Salmo salar). Dev Biol (Basel) 132,199-204.

Houston RD, CS Haley, A Hamilton, DR Guy, AE Tinch, JB Taggart, BJ McAndrew, SC Bishop. 2008 . Major quantitative trait loci affect resistance to infectious pancreatic necrosis in Atlantic salmon (Salmo salar). Genetics 178,1109-1115.

Kjøglum S, U Grimholt, S Larsen. 2005. Non-MHC genetic and tank effects influence disease challenge tests in Atlantic salmon (Salmo salar). Aquaculture 250, 102-109.

Kjøglum S, S Larsen, HG Bakke, U Grimholt. 2006. How specific MHC class I and class II combinations affect disease resistance against infectious salmon anaemia in Atlantic salmon (Salmo salar). Fish Shellfish Immunol 21, 431-441.

Klein J, C O'Huigin. 1994. The conundrum of nonclassical major histocompatibility complex genes. Proc Natl Acad Sci USA 91, 6251-6252.

Lande R, R Thompson. 1990. Efficiency of marker-assisted selection in the improvement of quantitative traits. Genetics 124, 743-756.

Langefors A, J Lohm, M Grahn, O Andersen, T von Schantz. 2001. Association between major histocompatibility complex class IIB 
alleles and resistance to Aeromonas salmonicida in Atlantic salmon. Proc $R$ Soc Lond B Biol Sci 268, 479-485.

Leal J, D Woywood. 2007. Piscirickettsiosis en Chile: Avances y perspectivas para su control. SalmoCiencia 2, 34-42.

Liu ZJ, JF Cordes. 2004. DNA marker technologies and their applications in aquaculture genetics. Aquaculture 238, 1-37.

Lohm J, M Grahn, Å Langefors, Ø Andersen, A Storset, T von Schantz. 2002. Experimental evidence for major histocompatibility complexallele-specific resistance to a bacterial infection. Proc $R$ Soc Lond B Biol Sci 269, 2029-2033.

Lund T, T Gjedrem, H Bentsen, D Eide, HJS Larsen, KH Roed. 1995. Genetic variation in immune parameters and associations to survival in Atlantic salmon. J Fish Biol 46, 748-758.

Martin S, S Blaney, D Houlihan, CJ Secombes. 2006. Transcriptome response following administration of a live bacterial vaccine in Atlantic salmon (Salmo salar). Mol Immunol 43, 1900-1911.

Martínez V, G Thorgaard, B Robison, M Sillanpää. 2005. Simultaneous consideration of environmental and genetic effects in QTL mapping: an application to early development in double haploid lines of rainbow trout. Genet Res 86, 209-221.

Martínez V. 2007. Marker-assisted selection in fish and shellfish breeding schemes. In: Marker-Assisted selection - Current status and future perspectives in crops, livestock, forestry and fish. FAO, Rome, Italy, Pp 329-362.

Meuwissen THE, B Hayes, M Goddard. 2001. Prediction of total genetic value using genomewide dense marker maps. Genetics 157 , 1819-1829.

Meuwissen THE, A Karlsen, S Lien, I Olsaker, ME Goddard. 2002. Fine mapping of a quantitative trait locus for twinning rate using combined linkage and linkage disequilibrium mapping. Genetics 161, 373-9.

Meuwissen THE. 2003. Genomic selection the future of marker-assisted selection and animal breeding. In: Marker-assisted selection: a fast track to increase genetic gain in plant and animal breeding? FAO, Rome, Italy.

Miller KM, RE Withler. 1996. Sequence analysis of a polymorphic MHC class II gene in Pacific salmon. Immunogenetics 43, 337-351.

Miller KM, JR Winton, AD Schulze, MK Purcell, TJ Ming. 2004. Major histocompatibility complex loci are associated with susceptibility of Atlantic salmon to infectious hematopoietic necrosis virus. Environ Biol Fishes 69, 307-316.

Moen T, KT Fjalestad, H Munck, L Gomez-Raya. 2004ª . A multistage testing strategy for detection of quantitative trait loci affecting disease resistance in Atlantic salmon. Genetics 167, 851-858.

Moen T, B Hoyheim, H Munck, L Gomez-Raya. 2004 . A linkage map of Atlantic salmon (Salmo salar) reveals an uncommonly large difference in recombination rate between the sexes. Anim Genet 35, 81-92.

Moen T, AK Sonesson, B Hayes, S Lien, H Munck, THE Meuwissen. 2007. Mapping of a quantitative trait locus for resistance against infectious salmon anaemia in Atlantic salmon (Salmo salar): comparing survival analysis with analysis on affected/resistant data. BMC Genetics 8, 53

Nichols KM, WP Young, RG Danzmann, BD Robison, C Rexroad, M Noakes, RB Phillips, P Bentzen, I Spies, K Knudsen, FW Allendorf, BM Cunningham, J Brunelli, H Zhang, S Ristow, R Drew, KH Brown, PA Wheeler, GH Thorgaard. 2003. A consolidated linkage map for rainbow trout (Oncorhynchus mykiss). Anim Genet 34, 102-115.

Ødegård J, I Olesen, B Gjerde, G Klemetsdal. 2006. Evaluation of statistical models for genetic analysis of challenge test data on furunculosis resistance in Atlantic salmon (Salmo salar): prediction of field survival. Aquaculture 259, 116-123.

Ødegård J, I Olesen, B Gjerde, G Klemetsdal. 2007. Evaluation of statistical models for genetic analysis of challenge-test data on ISA resistance in Atlantic salmon (Salmo salar): Prediction of progeny survival. Aquaculture 266, 70-76.

Ozaki A, T Sakamoto, S Khoo, K Nakamura, MRM Coimbra, T Akutsu, N Okamoto. 2001. Quantitative trait loci (QTLs) associated with resistance/susceptibility to infectious pancreatic necrosis virus (IPNV) in rainbow trout (Oncorhynchus mykiss). Mol Genet Genomics 265, 23-31.

Palti Y, JE Parsons, GH Thogaard. 1999. Identification of candidate DNA markers associated with IHN virus resistance in backcrosses of rainbow (Oncorhynchus mykiss) and cutthroat trout (O. clarki). Aquaculture 173, 81-94.

Palti Y, KM Nichols, KI Waller, JE Parsons, GH Thorgaard. 2001. Association between DNA polymorphisms tightly linked to MHC class II genes and IHN virus resistance in backcrosses of rainbow and cutthroat trout. Aquaculture 194, 283-289.

Perry GML, P Tarte, S Croisetière, P Belhumeur, L Bernatchez. 2004. Genetic variance and covariance for $0+$ brook charr (Salvelinus fontinalis) weight and survival time of furunculosis (Aeromonas salmonicida) exposure. Aquaculture 235, 263-271.

Phillips RB, KA Keatley, MR Morasch, AB Ventura, KP Lubieniecki, BF Koop, RG Danzmann, WS Davidson. 2009. Assignment of Atlantic salmon (Salmo salar) linkage groups to specific chromosomes: conservation of large syntenic blocks corresponding to whole chromosome arms in rainbow trout (Oncorhynchus mykiss). BMC Genet 10, 46.

Piepho HP, G Koch. 2000. Codominant analysis of banding data from a dominant marker system by normal mixtures. Genetics 155,14591468.

Pomp D, MF Allan, SR Wesolowski. 2004. Quantitative genomics: exploring the genetic architecture of complex trait predisposition. J Anim Sci 82, 300-312.

Purcell MK, KM Nichols, JR Winton, G Kurath, GH Thorgaard, P Wheeler, JD Hansen, RP Herwig, LK Park. 2006. Comprehensive gene expression profiling following DNA vaccination of rainbow trout against infectious hematopoietic virus. Mol Immunol 43, 2089-2106.

Qureshi ST, E Skamene, D Malo. 1999. Comparative genomics and host resistance against infectious diseases. Emerging Infect Dis 5, 36-47.

Rise ML, SRM Jones, GD Brown, KR von Schalburg, WS Davidson, BF Koop. 2004 . Microarray analyses identify molecular biomarkers of Atlantic salmon macrophage and hematopoietic kidney response to Piscirickettsia salmonis infection. Physiol Genomics 20, 21-35.

Rise ML, KR von Schalburg, GD Brown, RHDevlin, MA Mawer, N Kuipers, M Busby, M Beetz-Sargent, R Alberto, AR Gibbs, P Hunt, R Shukin, JA Zeznik, C Nelson, SRM Jones, DE Smailus, SJM Jones, JE Schein, MA Marra, YSN Butterfield, JM Stott, SH Ng, WS Davidson, BF Koop. $2004^{\mathrm{b}}$. Development and application of a salmonid EST database and cDNA microarray: data mining and interspecific hybridization characteristics. Genome Res 14, 478-490.

Rodriguez MF, S LaPatra, S Williams, T Famula, B May. 2004. Genetic markers associated with resistance to infectious hematopoietic necrosis in rainbow and steelhead trout (Oncorhynchus mykiss) backcrosses. Aquaculture 241, 93-115.

Røed KH, KT Fjalestad, A Strømsheim. 1993. Genetic variation in lysozyme activity and spontaneous haemolytic activity in Atlantic salmon (Salmo salar). Aquaculture 114, 19-31.

Rothschild MF, M Soller. 1997. Candidate gene analysis to detect genes controlling traits of economic importance in domestic livestock. Probe 8, 13-20.

Sakamoto T, RG Danzmann, K Gharbi, P Howard, A Ozaki, SK Khoo, RA Woram, N Okamoto, MM Ferguson, L Holm, R Guyomard, B Hoyheim. 2000. A microsatellite-based map for rainbow trout (Oncorhynchus mykiss) characterized by large sex-specific differences in recombination rates. Genetics 155, 1331-1345.

Salte R, HM Gjøen, K Nordberg, T Gjedrem. 1993. Plasma protein levels as potential marker traits for resistance to furunculosis. J Fish Dis 16, 561-568.

Sato A, F Figueroa, BW Murray, E Malaga-Trillo, Z Zaleska-Rutczynska, H Sultmann, S Toyosawa, C Wedekind, N Steck, J Klein. 2000. Nonlinkage of major histocompatibility complex class I and class II loci in bony fishes. Immunogenetics 51, 108-116. 
SERNAPESCA, Servicio Nacional de Pesca, Chile. 2007. Balance de la situación sanitaria de la anemia infecciosa del salmón en Chile de julio del 2007 a julio del 2008.

Shum BP, R Rajalingam, KE Magor, K Azumi, WH Carr, B Dixon, RJ Stet, MA Adkison, RP Hedrick, P Parham. 1999. A divergent non-classical class I gene conserved in salmonids. Immunogenetics 49, 479-490.

Shum BP, L Guethlein, LR Flodin, MA Adkison, RP Hedrick, RB Nehring, RJ Stet, C Secombes, P Parham. 2001. Modes of salmonid MHC class I and II evolution differ from the primate paradigm. J Immunol 166, 3297-3308.

Shum BP, PM Mason, KE Magor, LR Flodin, RJ Stet, P Parham. 2002. Structures of two major histocompatibility complex class I genes of the rainbow trout (Oncorhynchus mykiss). Immunogenetics 54, 193-199.

Smith P, J Larenas, P Vera, J Contreras, C Venegas, ME Rojas, A Guajardo. 2001. Principales enfermedades de los peces salmonídeos cultivados en Chile. Monografías de Medicina Veterinaria 21, 3-19.

Standal M, B Gjerde. 1987. Genetic variation in survival of Atlantic salmon during searearing period. Aquaculture 66, 197-207.

Stear MJ, SC Bishop, BA Mallard, H Raadsma. 2001. The sustainability, feasibility and desirability of breeding livestock for disease resistance. Res Vet Sci 71, 1-7.

Stet RJM, B de Vries, K Mudde, T Hermsen, J van Heerwaarden, BP Shum, U Grimholt. 2002. Unique haplotypes of co-segregating major histocompatibility class II A and class II B alleles in Atlantic salmon (Salmo salar) give rise to diverse class II genotypes. Immunogenetics 54, 320-331.

Thorgaard GH, GS Bailey, D Williams, DR Buhler, SL Kaattari, SS Ristow, JD Hansen, JR Winton, JL Bartholomew, JJ Nagler, PJ Walsh, MM Vijayan, RH Devlin, RW Hardy, KE Overturf, WP Young, BD Robison, C Rexroad, Y Palti. 2002. Status and opportunities for genomics research with rainbow trout. Comp Biochem Physiol B Biochem Mol Biol 133, 609-646.

Tsoi SC, JM Cale, IM Bird, KV Ewart, LL Brown, SE Douglas. 2003. Use of human cDNA microarrays for identification of differentially expressed genes in Atlantic salmon liver during Aeromonas salmonicida infection. Mar Biotechnol 5, 545-554.

Tsoi SC, KV Ewart, S Penny, K Melville, RS Liebscher, LL Brown, SE Douglas. 2004. Identification of immune-relevant genes from Atlantic salmon using suppression subtractive hybridization. Mar Biotechnol 6, 199-214.

Villanueva B, R Pong-Wong, JA Woolliams. 2002. Marker assisted selection with optimised contributions of the candidates to selection. Genet Sel Evol 34, 679-703.

von Schalburg KR, ML Rise, GA Cooper, GD Brown, RA Gibbs, CC Nelson, WS Davidson, BF Koop. 2005. Fish and Chips: Various methodologies demonstrate utility of a 16,006-gene salmonid microarray. BMC Genomics 56, 126.

Walsh B, D Henderson. 2004. Microarrays and beyond: what potential do current and future genomics tools have for breeders? J Anim Sci 82, 292-299.

Withler RE, PT Evelyn. 1990. Genetic variation in resistance to bacterial kidney disease within and between two strains of coho salmon from British Columbia. Trans Am Fish Soc 119, 1003-1009.

Woram RA, C McGowan, JA Stout, K Gharbi, MM Ferguson, B Hoyheim, EA Davidson, WS Davidson, CE Rexroad, RG Danzmann. 2004. A genetic linkage map for Arctic char (Salvelinus alpinus): evidence for higher recombination rates and segregation distortion in hybrid versus pure strain mapping parents. Genome 47, 304-315.

Young WP, PA Wheeler, VH Coryell, P Keim, GH Thorgaard. 1998. A detailed linkage map of rainbow trout produced using doubled haploids. Genetics 148, 839-850. 